\title{
Uso del ensayo de implante y la técnica de emisión acústica para estudiar el agrietamiento asistido por hidrógeno en la zona fundida en la soldadura de un acero HSLA-80 ${ }^{(\bullet)}$
}

\begin{abstract}
H.C. Fals ${ }^{(*)}$ y R.E. Trevisan ${ }^{(* *)}$
Resumen Se estudió el agrietamiento asistido por hidrógeno en la zona fundida, usando dos alambres tubulares (E 70T-5 y E $120 \mathrm{~T} 5-\mathrm{K} 4$ ) y una mezcla gaseosa de $\mathrm{CO}_{2}+5 \% \mathrm{H}_{2}$, para inducir altos niveles de hidrógeno difusible en la soldadura de un acero HSLA-80. Un sistema de medición de emisión acústica basado en un voltímetro RMS (Root Mean Square), acoplado a la instalación de ensayo de implante (NF 89-100), permitió determinar la energía, amplitud RMS y número de ciclos de la señal. Se observó por microscopía óptica y electrónica de barrido, que en todos los ensayos las grietas se formaron en la zona parcialmente fundida. Cuando se usó el alambre E 70T-5, las grietas se propagaron a través de la región de crecimiento de grano de la zona térmicamente afectada, predominando el modo de fractura cuasiclivage. En los ensayos del alambre E 120 T5-K4, las grietas crecieron verticalmente a través de la zona fundida, verificándose mayor incidencia de un modo de fractura mixto. Se encontró una relación importante entre los parámetros de la señal de EA y los modos de fractura que actúan en el mecanismo de agrietamiento.
\end{abstract}

Palabras clave: Aceros microaleados. Soldadura. Agrietamiento asistido por hidrógeno. Modos de fractura. Emisión acústica.

\section{Implant test and acoustic emisssion technique used to investigate hydrogen assisted cracking in the melted zone of a welded HSLA-80 steel}

\begin{abstract}
Weld metal hydrogen assisted cracking was studied using two flux cored wire (AWS E 70T-5 and AWS E $120 \mathrm{~T} 5-\mathrm{K} 4$ ) and a mixture gas of $\mathrm{CO}_{2}+5 \% \mathrm{H}_{2}$ to induce high values of diffusible hydrogen in high strength low alloy steel (HSLA-80) weldments. An Acoustical Emission Measurement System (AEMS) RMS voltmeter was coupled to the implant test (NF 89-100) apparatus to determine energy, amplitude and event numbers of signal. All cracks were initiated in the partially melted zone and propagated into the coarse-grained region of the heat affected zone when E 70 T5 consumable was used, and the quasi-cleavage fracture mode was predominant. When E 120 T5 K4 consumable was used the cracks propagated vertically across the fusion zone, and a mixed fracture mode was the most important. A significant relationship between acoustic emission parameters and fracture modes was found.
\end{abstract}

Keywords: Microalloy steel. Welding. Hydrogen assisted cracking. Fracture modes. Acoustic emission.

(•) Trabajo recibido el día 22 de enero de 1999 y aceptado en su forma final el 19 de agosto de 1999.

(*) Universidad de Oriente. Facultad de Eng. Mecánica. Av. Las Américas, Santiago de Cuba 90400 (Cuba).

(**) Universidade Estadual de Campinas. DEF/FEM. Baräo Geraldo. Campinas. 13083-970 CP 6122. SP (Brasil). 


\section{INTRODUCCIÓN}

Los aceros de alta resistencia y baja aleación han alcanzado en las últimas décadas una creciente aplicación industrial debido a su excelente combinación de altas propiedades mecánicas y soldabilidad. No obstante, el fenómeno de agrietamiento asistido por hidrógeno causado en las uniones soldadas de este material continúa ocupando un lugar destacado, en los problemas metalúrgicos, en la soldadura de estos aceros.

El agrietamiento en frío, como también es conocido, ha dejado de ocurrir con mayor frecuencia en la zona afectada térmicamente (ZAT) de la unión soldada y cada vez más es típico de la zona fundida (1), presentando por tanto nuevas características que deben analizarse para entender correctamente el problema.

Además de la tensión de restricción, la microestructura y el nivel de hidrógeno, el aumento del agrietamiento causado por hidrógeno en la zona fundida (ZF) se debe, fundamentalmente, al incremento de la temperatura de transformación de los aceros de baja aleación, provocado por el bajo contenido de carbono y elementos aleantes (2). Esto, a su vez, trae como consecuencia que la transformación austenitaferrita de la ZAT, ocurra antes de la transformación de la ZF y, por tanto, aumenta en concentración de hidrógeno en la región, debido a que la difusión de hidrógeno de la ZF hacia la ZAT disminuye.

El análisis de la forma de propagación de las grietas es importante para entender las características del fenómeno. Beachem (3) expuso la teoría de la microplasticidad para explicar el fenómeno del agrietamiento por hidrógeno y propuso un diagrama que relaciona los modos de fractura con el coeficiente de concentración de tensión en el modo I $\left(K_{\mathrm{I}}\right)$.

Este diagrama fue usado por Yurioka et al. (4) para analizar los resultados de ensayos de implante, lo que les llevó a ponerse de acuerdo en explicar que el modo de fractura por microcoalescencia de vacíos (MCV), actúa, predominantemente, para elevados valores de tensiones aplicadas, y en la medida que la tensión disminuye, aparecen los modos por cuasiclivage (CC) e intergranular (IG). No obstante, se observó en el trabajo publicado por Gedeon et al. (5), que para mayores niveles de hidrógeno que los usados para obtener el modelo de Beachem, los modos IG y CC estuvieron presentes con altos niveles de tensión aplicada.

Debido a la importancia del agrietamiento y fallas en las uniones soldadas, se han utilizado varias técnicas para su detección; no obstante, la técnica de emisión acústica (EA) ha tenido gran aceptación por su versatilidad, bajo costo y viabilidad para la determinación de fenómenos en tiempo real.
Esta técnica viene estudiándose desde los años 60 , y se utiliza, principalmente, como herramienta de monitorización de estructuras mecánicas en ensayos no destructivos (crecimiento de grietas). Se han realizado varias investigaciones para detectar la formación y crecimiento de grietas durante y después de la soldadura, o en piezas en servicio, usando la técnica de EA (6).

Como la EA se genera como producto de los cambios en el material, en el dominio de la deformación y tensión, el análisis de la forma de onda de la EA facilita la obtención de información sobre el aspecto dinámico del fenómeno que la origina (7). Las características de la señal eléctrica de la EA dependen del mecanismo que la produjo, del material por donde se propaga y del sensor usado.

A pesar de la amplia aplicación de la técnica de $\mathrm{EA}$, se encontraron pocos trabajos que relacionen la extensión y el tipo de modo de fractura que ocurren en el agrietamiento asistido por hidrógeno con la amplitud RMS, energía y número de ciclos medidos de la señal ( 8 y 9). Esta correlación puede ser importante para conocer, a través del análisis de la señal de EA, el tipo de micromecanismo que está actuando en la fractura de un material, y de cierta forma adecuar correctamente las medidas a tomar para evitar fallas catastróficas.

Este trabajo estudia el agrietamiento asistido por hidrógeno en la ZF de las uniones soldadas de un acero microaleado, empleando el ensayo de implante modificado, además de emplear la técnica de EA para caracterizar los modos de fractura que actúan durante el agrietamiento de las probetas

\section{MATERIALES Y MÉTODO EXPERIMENTAL}

Para el desarrollo del trabajo se utilizó un acero microaleado, con la composición química y propiedades mecánicas que se muestran en la tabla I. El proceso de soldadura con alambre tubular (Flux cored arc welding-FCAW), se usó con aporte térmico constante, equivalente a $0,659 \mathrm{~kJ} / \mathrm{mm}$. Los alambres fueron del tipo AWS E 70T-5 (T-75) y E 120 T5-K4 (T-120). Las composiciones químicas de los depósitos obtenidos con cada alambre se muestran en la tabla II. $\mathrm{Al} \mathrm{CO}_{2}$ usado como gas de protección, se le añadió $5 \% \mathrm{H}_{2}$ para inducir altos valores de hidrógeno difusible en la unión soldada.

Para determinar el nivel de hidrógeno difusible, se empleó la técnica de cromatografia gaseosa, de acuerdo con la norma ANSI/AWS A4.3-86 (10). Los resultados mostraron que el nivel de hidrógeno fue diferente para cada alambre usado, siendo como media $22,37 \mathrm{ml} / 100 \mathrm{~g}$ del metal depositado (MD), para el alambre T-120 y $18,02 \mathrm{ml} / 100 \mathrm{~g}$ MD para el alambre T-75. 
TABLA I.- Composición química (\% en peso) y propiedades mecánicas del acero usado

TABLE I.- Chemical composition (wt \%) and mechanical properties of the steel

\begin{tabular}{|c|c|c|c|c|c|c|c|c|c|c|c|}
\hline $\mathrm{C}$ & $\mathrm{Mn}$ & $\mathrm{Ni}$ & $\mathrm{Cr}$ & $\mathrm{Si}$ & $\mathrm{Cu}$ & $\mathrm{P}$ & $\mathrm{S}$ & $\mathrm{Mo}$ & $\mathrm{V}$ & $\mathrm{Ti}$ & $\mathrm{Al}$ \\
\hline 0,12 & 0,99 & 0,015 & 0,52 & 0,20 & 0,26 & 0,03 & 0,007 & 0,34 & 0,029 & 0,007 & 0,026 \\
\hline \multicolumn{3}{|c|}{$\begin{array}{c}\text { Límite elástico } \\
\text { convencional }\left(R_{\mathrm{e} 0,2}\right) \\
\mathrm{MPa}\end{array}$} & $\begin{array}{c}\text { Máxima resistencia } \\
\text { a la tracción }\left(R_{\mathrm{m}}\right), \\
\mathrm{MPa}\end{array}$ & \multicolumn{3}{c|}{$\begin{array}{c}\text { Alargamiento }(A r), \\
\%\end{array}$} & \multicolumn{3}{c|}{ Dureza $\left(\mathrm{HV}_{10}\right)$} \\
\hline \multicolumn{3}{|c|}{805} & \multicolumn{3}{c|}{19,06} & & 275 \\
\hline
\end{tabular}

TABLA II.- Composición química (\% en peso) en los depósitos de los alambres tubulares

TABLE II.-Chemical composition (wt \%) of weld deposit

\begin{tabular}{|l|c|c|c|c|c|c|c|}
\hline Alambre & $\mathrm{C}$ & $\mathrm{Mn}$ & $\mathrm{Si}$ & $\mathrm{Ni}$ & $\mathrm{Cr}$ & $\mathrm{Mo}$ & $\mathrm{Nb}$ \\
\hline E 70T-5 & 0,07 & $1,42-1,66$ & $0,46-0,6$ & 0,02 & 0,03 & 0,01 & 0,01 \\
E120 T5-K4 & 0,07 & 1,73 & 0,46 & 2,36 & 0,52 & 0,45 & 0,01 \\
\hline Alambre & $\mathrm{Ti}$ & $\mathrm{V}$ & $\mathrm{Al}$ & $\mathrm{P}$ & $\mathrm{S}$ & $\mathrm{N}(\mathrm{ppm})$ & $\mathrm{O}(\mathrm{ppm})$ \\
\hline E 70T-5 & 0,01 & $<0,01$ & $<0,01$ & 0,013 & 0,014 & $55-42$ & $542-439$ \\
E120 T5-K4 & 0,01 & $<0,01$ & $<0,01$ & 0,021 & 0,008 & 145 & 385 \\
\hline
\end{tabular}

Fuente: Valores cedidos por los fabricantes.

Tomando como base la norma NF 89-100 (Cold Cracking Implant Test) (11), que detalla el procedimiento del ensayo de soldabilidad conocido como ensayo de implante, fueron introducidas algunas modificaciones en los parámetros del ensayo, para provocar el agrietamiento causado por hidrógeno en la zona fundida de la unión soldada y no en la ZAT como es lo previsto. Las modificaciones fueron las siguientes:

- La probeta-implante fue realizada sin entalla para evitar la formación del agrietamiento en la ZAT.

- Durante la solidificación de la zona fundida, se inyectó neumáticamente una pastilla aguda de cerámica, para obtener triaxialidad de tensiones, similar a un entalle en la región.

- El orificio que sirve de alojamiento de la probeta-implante en la chapa base, fue $1 \mathrm{~mm}$ mayor en su diámetro que el diámetro externo del implante, para así aumentar la concentración de tensiones en la intercepción con la zona fundida, chapa base e implante;

- Fue mecanizada una ranura en la chapa base, simétrica al orificio de localización del implante, para disminuir la dilución del metal depositado. Los ensayos se realizaron usando tres niveles de tensión, equivalentes al 66, 57 y $52 \%$ del límite elástico $\left(R_{\mathrm{e} 0,2}\right)$ de cada tipo de metal depositado.
De esta forma, los valores usados corresponden a 580,501 y $457 \mathrm{MPa}$ en el alambre T-120 y 330,285 y $260 \mathrm{MPa}$ para el alambre T-75.

\subsection{Sistema de medición de emisión acústica (SMEA)}

En la figura 1 se muestra, de forma representativa, un diagrama de bloques, que fue acoplado a la instalación del ensayo de implante, permitiendo monitorizar la formación de las grietas asistidas por hidrógeno. Los parámetros de la configuración usada del SMEA fueron:

- Sensor de emisión acústica. WD-A23 de banda amplia, sensibilidad media de $-73 \mathrm{~dB}$ referido a $1 \mathrm{~V} / \mu$ bar. Amplitud de frecuencia de 100 a $1.000 \mathrm{kHz}$.

- Filtro preamplificador. Modelo 1220A de 100$300 \mathrm{kHz}$. Ganancia de $40 \mathrm{~dB}$.

- Voltímetro RMS. Constante de tiempo en la salida de la señal de $0,10 \mathrm{~ms}(10 \mathrm{kHz})$.

- Tarjeta CAD12/36 con fecuencia de adquisición de $100 \mathrm{kHz}$ vía acceso directo a la memoria (DMA) y ajuste del nivel de referencia $(0,025$ V).

- Microcomputador PC - Pentium. $133 \mathrm{MHz}, 32$ $\mathrm{Mb}$ de memoria RAM, 2,1 Gb de disco duro. 


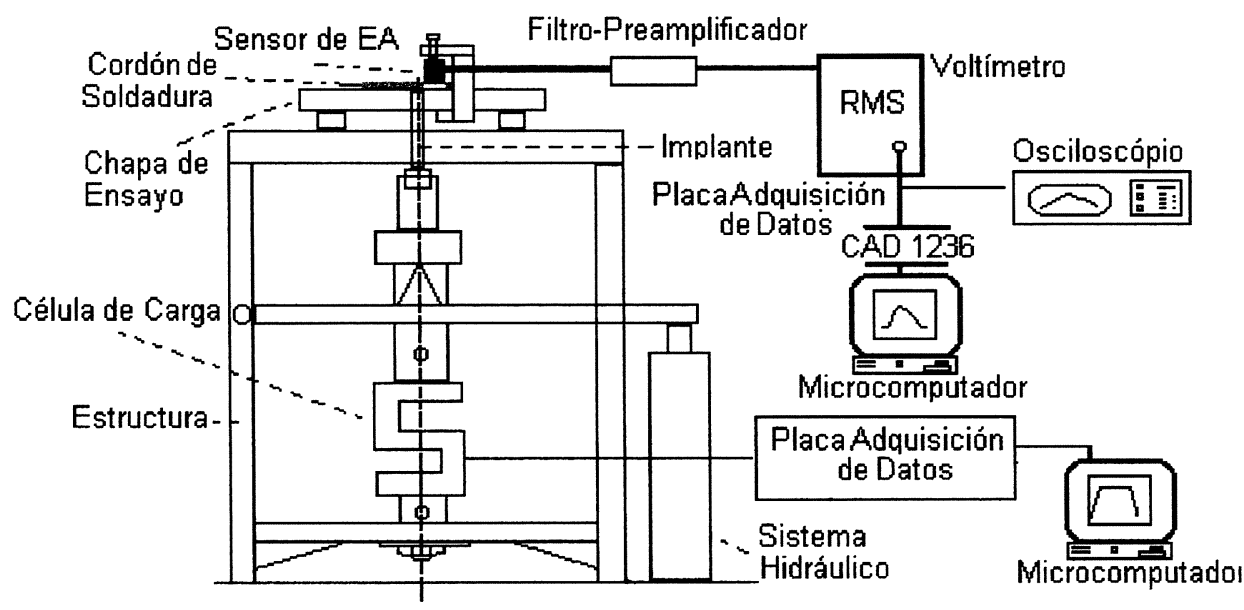

Fig. 1.- Representación de la instalación de ensayo de implante con los sistemas de monitorización de fuerza y EA.

FIG. 1.- Experimental set-up for monitoring AE and force signal in implant test.

- Software RUPTURA para la adquisición de los datos de emisión acústica con frecuencia de adquisición de $50 \mathrm{kHz}$.

A través del análisis de la forma de las ondas de $\mathrm{EA}$, se midieron los siguientes parámetros: número de ciclos, energía y amplitud RMS de la señal de EA. El sumatorio de los tres parámetros fueron relacionados con los modos de fractura para cada caso.

Las técnicas de microscopía óptica, microscopía electrónica de barrido y análisis de imagen, se usaron también para estudiar la microestructura, los lugares de formación y propagación de las grietas, así como para medir las áreas correspondientes a cada micromecanismo de fractura.

\section{RESULTADOS Y DISCUSIÓN}

En los ensayos de implante realizados, independientemente del tipo de alambre, las grietas asistidas por hidrógeno siempre se formaron en la región de alta concentración de tensiones, localizada aproximadamente en la línea de fusión, exactamente en el lugar donde fue provocado (en la nueva propuesta de ensayo) un cambio brusco de sección por causa del aumento de la tolerancia entre el implante y la chapa base.

No obstante, se observó que el sentido de propagación de las grietas dependió directamente del tipo de alambre usado en cada ensayo. Esto significa que las grietas crecieron en diferentes direcciones de la unión soldada, de acuerdo con las propiedades mecánicas y microestructurales de la zona fundida.

En los ensayos realizados con el alambre T-75, se observó que, de forma general, las grietas se propagaron transgranular e intergranularmente en la dirección de la zona de crecimiento de grano de la ZAT (CGZAT), como se observa en la figura 2.

En esta zona, como resultado del ciclo térmico impuesto en la soldadura, la austenita primaria sufrió un gran crecimiento de grano y se formó martensita (dureza media: $30 \mathrm{HRc}$ ), susceptible a la propagación de las grietas.

Además del aumento de la fragilidad que ocurre en la CGZAT, el depósito obtenido con el alambre T-75, también desempeñó un papel importante en la determinación de la dirección de propagación de las grietas, ya que este depósito tuvo como característica fundamental una dureza inferior (24 HRc) a la dureza de la CGZAT. Es importante señalar que la ZF obtenida con este alambre estaba compuesta por

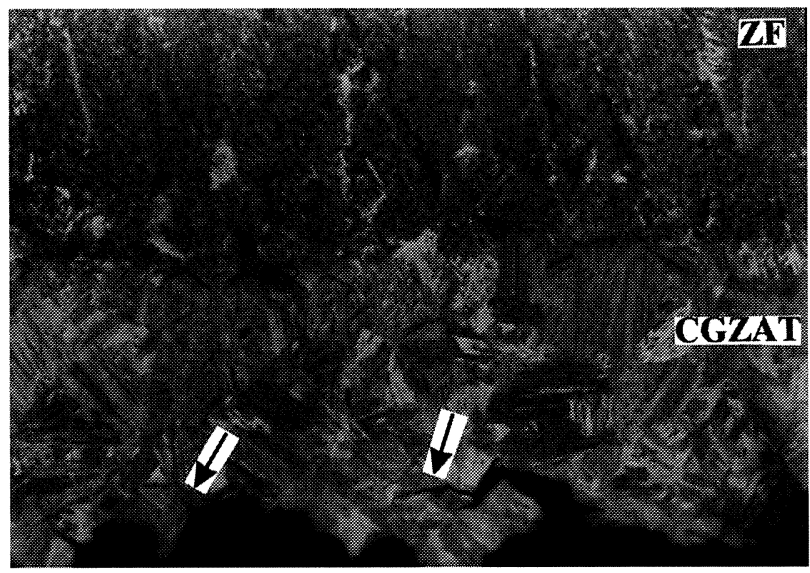

FIG. 2.- Propagación de las grietas intergranular y transgranularmente en la CGZAT. Alambre T-75. Nital $2 \% . \times 800$.

FIG. 2.- Intergranular and transgranular crack propagation in the CGHAZ. T-75 wire. Nital $2 \%$. $800 \times$. 
microestructuras de ferrita con diferentes morfologías tales como: ferrita de contornos de grano $(\mathrm{PF}[\mathrm{G}])$, ferrita poligonal intragranular $\left(\mathrm{PF}\left[\mathrm{I}_{\mathrm{FTI}}\right]\right)$, ferrita de segunda fase alineada (FS[A]) y ferrita acicular (AF), las cuales provocan la formación de una región tenaz más resistente al crecimiento de las grietas. En la figura 3 se muestra esta composición microestructural.

En los ensayos realizados usando el alambre T120 , las grietas se propagaron verticalmente en la dirección de la zona fundida, siendo transgranulares e intergranulares en el sentido de los grandes granos columnares, característicos de la región. Los depósitos obtenidos con el alambre T-120 se compusieron por microestructuras de martensitas y bainitas finas con dureza media de $35 \mathrm{HRc}$, superior a la dureza de la CGZAT. Las características microestructurales y la dureza de la zona fueron favorables para la propagación vertical de las grietas en los ensayos realizados con este alambre.

\subsection{Tiempos de falla $\left(T_{\mathrm{f}}\right)$ y tensión crítica de falla $\left(\sigma_{\mathbf{c}}\right)$}

Los ensayos de implante realizados con el alambre $\mathrm{T}-75$ presentan una rotura para $\sigma=330 \mathrm{MPa}$, equivalente al $66 \%$ del límite elástico $\left(R_{\mathrm{e} 0,2}\right)$ del depósito, o sea, la máxima tensión aplicada en los ensayos para esta condición. El tiempo medio de falla $\left(T_{\mathrm{f}}\right.$, en esta condición experimental fue de $28 \mathrm{~min}$.

En los ensayos realizados con el alambre T-120, las probetas fallaron siguiendo la representación gráfica de la figura 4. Puede observarse que los $T_{\mathrm{f}}$ aumentaron en la medida que la tensión aplicada fue menor, como era esperado y presentado por diversos trabajos en este campo.

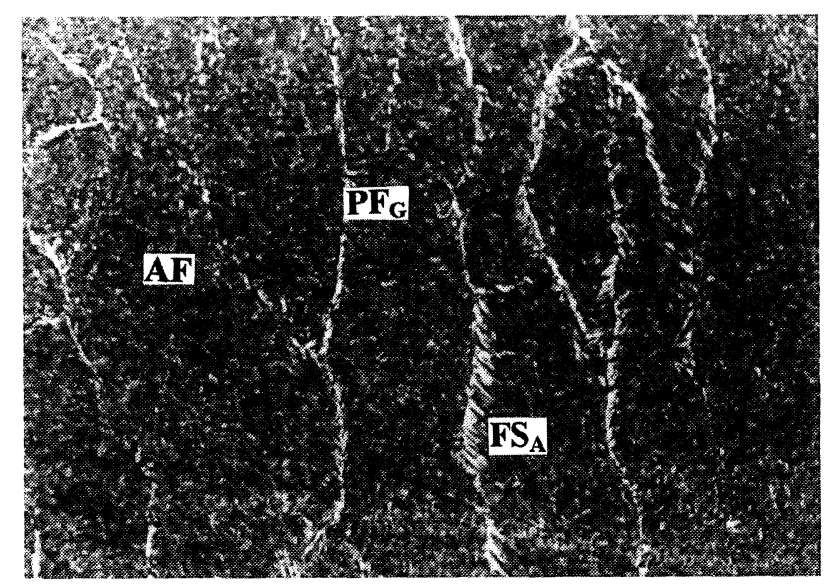

FIG. 3.- Microestructura de la zona fundida. Alambre T-75. Nital $2 \% . \times 1.000$.

FIG. 3.- Fusion zone microstructure obtained with T-75 wire. Nital $2 \% .1 .000 \times$.

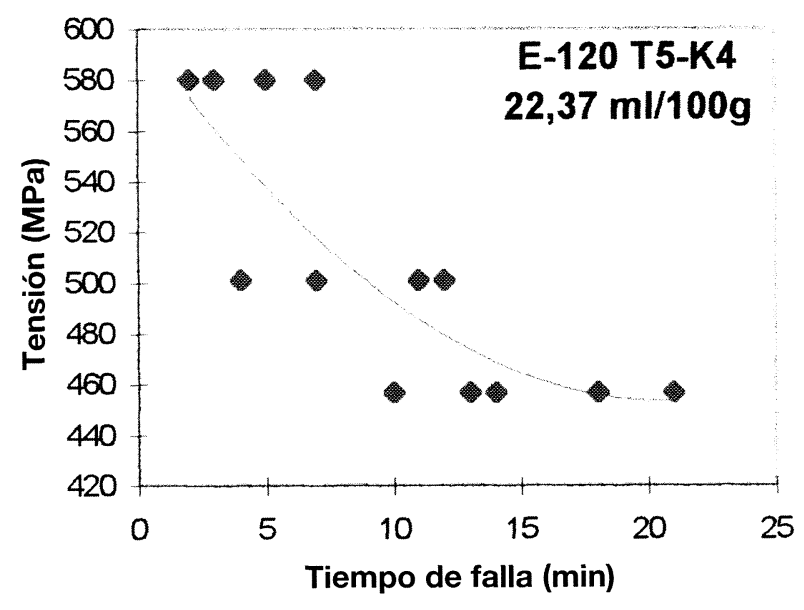

FIG. 4.- Tensión frente a tiempos de falla. Alambre $\mathrm{T}-120$.

FIG. 4.- Stress vs time to failure. T-120 wire.

Analizando la figura 4, observamos claramente que los $T_{\mathrm{f}}$ siempre fueron menores cuando se comparan con los resultados obtenidos en los experimentos realizados con el alambre T-75. Esto se origina fundamentalmente por dos factores importantes: la microestructura martensítica, más frágil, y el mayor nivel de hidrógeno difusible obtenido en el depósito del alambre T-120.

Como establece la norma NF 89-100, la tensión para la cual no ocurre la falla en el ensayo de implante durante $16 \mathrm{~h}$ se denomina tensión crítica de falla $\left(\sigma_{c}\right)$, valor que fue determinado para los alambres usados. En los ensayos realizados con el alambre T-75, la $\sigma_{\mathrm{c}}$ fue de $285 \mathrm{MPa}$, equivalente al $57 \%$ del límite elástico del depósito; ya en los ensayos realizados con el alambre T-120, este valor fue de $327 \mathrm{MPa}$ (33\% del límite elástico). De esta forma, se comprobó que el alambre T-120 fue más susceptible al fenómeno de agrietamiento asistido por el hidrógeno.

\subsection{Micromecanismos o modos de fractura}

A través de la técnica de microscopía electrónica de barrido, se observó que todas las superficies de fractura presentaron una morfología de fractura compleja, caracterizada por la coexistencia de los modos de fractura intergranular (IG), microcoalescencia de vacíos (MCV) y cuasiclivage (CC), y que estos variaron su extensión dependiendo de la microestructura presente en la zona de propagación de la grieta y de la tensión aplicada en los ensayos.

En los ensayos realizados con el alambre T-75, donde las grietas se propagaron en la CGZAT, fue predominante el modo $\mathrm{CC}$, como puede observarse en la tabla III, ocupando como media el 64,6\% del área total de la superficie de fractura; el modo 
TABLA III.- Correlación entre los modos de fractura que actúan en la zona fundida y la tensión aplicada TABLA III.-Relation between stress and fracture modes in the fusion zone

\begin{tabular}{|c|c|c|c|c|c|c|}
\hline \multicolumn{3}{|c|}{ Área media de fractura (\%) T-120 } & \multicolumn{4}{|c|}{ Área media de fractura $(\%) \mathrm{T}-75$} \\
\hline Tensión MPa \% $\operatorname{Re}_{0,2}$ & $\mathrm{MCV}$ & Mixta & Tensión, $\mathrm{MPa}\left(\% \operatorname{Re}_{0,2}\right)$ & $\mathrm{CC}$ & $\mathrm{MCV}$ & Mixta \\
\hline $580(66)$ & 15,7 & 73,6 & $330(66)$ & 64,6 & 11,4 & 23,9 \\
\hline $501(57)$ & 22,4 & 70,7 & $285(57)$ & - & - & - \\
\hline $457(52)$ & 35,4 & 60,2 & $260(52)$ & - & - & - \\
\hline \multicolumn{7}{|c|}{ Composición media del área de fractura mixta (\%). Alambre T-120 } \\
\hline Tensión MPa $\left(\% R e_{0,2}\right)$ & \multicolumn{2}{|c|}{$\mathrm{MCV}+\mathrm{CQ}$} & $\mathrm{IG}+\mathrm{MCV}+\mathrm{CQ}$ & \multicolumn{2}{|c|}{$\mathrm{IG}+\mathrm{MCV}$} & $\mathrm{IG}+\mathrm{CQ}$ \\
\hline $580(66)$ & \multicolumn{2}{|c|}{2,9} & 15,0 & \multicolumn{2}{|c|}{ - } & 55,7 \\
\hline $501(57)$ & \multicolumn{2}{|c|}{ - } & 7,8 & \multicolumn{2}{|c|}{22,1} & 40,8 \\
\hline $457(52)$ & \multicolumn{2}{|c|}{-} & - & \multicolumn{2}{|c|}{24,4} & 35,8 \\
\hline
\end{tabular}

MCV alcanzó $11,4 \%$ y también fue verificada la formación de zonas de fractura con morfología mixta, compuesta por los modos MCV, CC e IG, $23,9 \%$, los cuales actuaron en regiones muy pequeñas, imposibles de cuantificar separadamente.

La gran área del modo CC, en la superficie de fractura de los ensayos realizados con el alambre T75 , fue causada por la formación martensitica asociada a la CGZAT, donde las grietas se propagaron, y también por la elevada concentración de hidrógeno difusible usada en los experimentos para inducir el agrietamiento.

Los experimentos realizados con el alambre T-120 permitieron establecer una correlación entre los modos de fractura que actuaron en la zona fundida y la tensión aplicada en los ensayos. Como se observa en la tabla III, cuando la tensión fue aumentada en los ensayos, las áreas correspondientes al modo de fractura por MCV disminuyen, este resultado entra en contradicción con el modelo de microplasticidad de Beachen (3), usado para explicar el desarrollo del fenómeno de agrietamiento asistido por hidrógeno.

Este resultado fue originado por el elevado nivel de hidrógeno usado en los experimentos y por las características de solidificación y microestructurales de la ZF. Gedeon et al. (5) observaron resultados semejantes, proponiendo una nueva modificación al modelo de microplasticidad para niveles de hidrógeno mayores a los utilizados por Beachem (3). El trabajo reflejó que los modos de fractura IG y CC actúan, para altos valores de tensiones aplicadas, inhibiendo la formación del modo MCV.

En realidad, el alto nivel de hidrógeno usado provoca que la zona plástica en la punta de la grieta disminuya de tamaño, ya que los átomos de hidró- geno actúan como lugares de concentración de dislocaciones (12) impidiendo el crecimiento de la deformación plástica y con ello la coalescencia de microvacíos en la región. También la presencia de discontinuidades características de la $\mathrm{ZF}$, como inclusiones, precipitados, microporosidades, etc. pueden actuar como zonas de concentración de hidrógeno y generar la formación de microgrietas que pueden dar origen a las zonas de fractura mixta, caracterizadas por modos con pequeños grados de deformación plástica como CC e IG.

En la tabla III se observa que las áreas de fractura denominadas como mixtas tienen una marcada presencia en las superficies de fractura de los implantes ensayados con el alambre T-120 y estas aumentan en la medida que se incrementa la tensión aplicada. Pudo constatarse cómo fueron clasificadas las zonas de fractura mixtas y los modos presentes en cada área, notándose que los modos que más incidieron en el surgimiento de esta morfología fueron CC e IG, corroborando la incidencia de la microestructura y las discontinuidades en la formación de las áreas de fractura mixta.

\subsection{Análisis de las señales de EA}

Los resultados del análisis de las señales de EA permitieron establecer una importante correlación entre los principales parámetros de la señal (número de ciclos, energía y amplitud RMS) y los modos de fractura que operaron durante la propagación de las grietas, en cada condición experimental.

Todas las señales de EA analizadas presentaron como característica general la formación de varios ciclos de pequeña amplitud, antes de la fractura 
final de las probetas, indicando la existencia de alguna deformación plástica durante el proceso de fractura. Este fenómeno es típico del agrietamiento asistido por hidrógeno en uniones soldadas, donde la fractura ocurre a través de la combinación de los modos IG, CC y MCV.

En los experimentos realizados con el alambre T-75, se verificó que el número de ciclos, la energía y la amplitud RMS de la señal de EA fueron menores que en los experimentos realizados con el alambre T-120, independiente de la tensión aplicada, como se observa en la tabla IV. Este resultado fue debido a la diferencia entre los micromecanismos de fractura que actuaron en cada condición experimental.

La fractura tuvo como característica principal mayor incidencia del modo $\mathrm{CC}$, lo que provocó menor cantidad de ciclos en la señal de EA. Este modo de fractura envuelve poca deformación plástica en el material, la cual es la fuente principal de EA.

El aumento de los parámetros de la señal de EA, en los experimentos realizados con el alambre T120 , probablemente se debió a la formación de las zonas de fractura mixtas, características de la región fundida. Estas zonas de fractura fueron originadas, entre otras causas, por la formación de varias microgrietas formadas en las zonas de concentración de hidrógeno, provocando el aumento del número de ciclos de la señal.

En los experimentos realizados con el alambre T-120, se constató un aumento de la actividad acústica de acuerdo con la disminución de la tensión aplicada. Este resultado puede ser verificado mediante el análisis de los resultados presentados en la tabla IV.

Con el objetivo de facilitar la comprensión del efecto de la tensión aplicada en los parámetros de la señal de EA, se realizará un análisis comparativo entre los resultados de dos de las condiciones experimentales estudiadas.

En la figura 5(a), se observa parte de la señal de EA instantes antes de la fractura final de la probeta; en esta se constatan 4 ciclos de menor amplitud; la figura es representativa de un ensayo realizado con $580 \mathrm{MPa}$. En la figura 5(b) se muestra la superficie de fractura, mediante MEB, que generó la señal, observándose la formación de una gran área de fractura con morfología mixta y un área menor caracterizada como MCV.

En la figura 6(a), se muestra la parte de la señal de EA correspondiente al mismo instante de tiempo expuesto en la figura 5(a), con la diferencia de que en este caso se aplicaron $457 \mathrm{MPa}$ de tensión. En esta figura puede observarse que el número de ciclos es mayor (9). También, en correspondencia con el aumento del número de ciclos, existió un marcado aumento en el área del modo de fractura por MCV, como se observa cuando se comparan las macrografías mostradas en las figura 6(b) y figura 5(b).

Este análisis comparativo demuestra que existe una fuerte dependencia entre el número de ciclos de la señal de EA y la extensión en el área del modo de fractura por MCV, o sea, se constató que en la medida que aumentaba el área de fractura por MCV era mayor el número de ciclos, como consecuencia de la deformación plástica que ocurre en el material, producto de la coalescencia de microvacíos.

Como se observa en la figura 7 y en la tabla IV, la energía y amplitud RMS de la señal aumentaron cuando la tensión aplicada fue menor, es decir, cuando se incrementó el área de fractura por MCV. Este resultado debe ser aclarado, ya que como se discute en la literatura consultada (3-5), los ciclos de EA característicos del modo de fractura MCV poseen baja amplitud y energía, lo que también se comprobó en el desarrollo del trabajo. En este punto surge una contradicción con respecto a los resultados mostrados en la figura 7.

No obstante, en este trabajo fueron determinados la amplitud RMS y energía total de la señal y no de los ciclos individuales; por tanto, los valores de estos parámetros de la señal que se exponen correspoden al sumatorio de todos los ciclos que ocurren durante el proceso de fractura en los ensayos.

TABLA IV.- Correlación entre la tensión aplicada y los parámetros de la señal de EA

TABLE IV.- Relation between stress and AE parameters

\begin{tabular}{|c|c|c|c|c|}
\hline Alambres & $\begin{array}{c}\text { Tensión aplicada, } \\
\text { MPa } \\
\left(\% R_{\mathrm{e} 0,2}\right.\end{array}$ & $\begin{array}{c}\text { Número medio } \\
\text { de ciclos }\end{array}$ & $\begin{array}{c}\text { Energía media } \\
\left(\mathrm{V}^{2} \mathrm{~s}\right)\end{array}$ & $\begin{array}{c}\text { Amplitud RMS } \\
\text { media (V) }\end{array}$ \\
\hline T-75 & $330(66)$ & 3 & 0,069 & 6,82 \\
T-120 & $580(66)$ & 9 & 0,108 & 8,28 \\
T-120 & $501(57)$ & 12 & 0,113 & 9,95 \\
T-120 & $457(52)$ & 19 & 0,126 & 12,94 \\
\hline
\end{tabular}



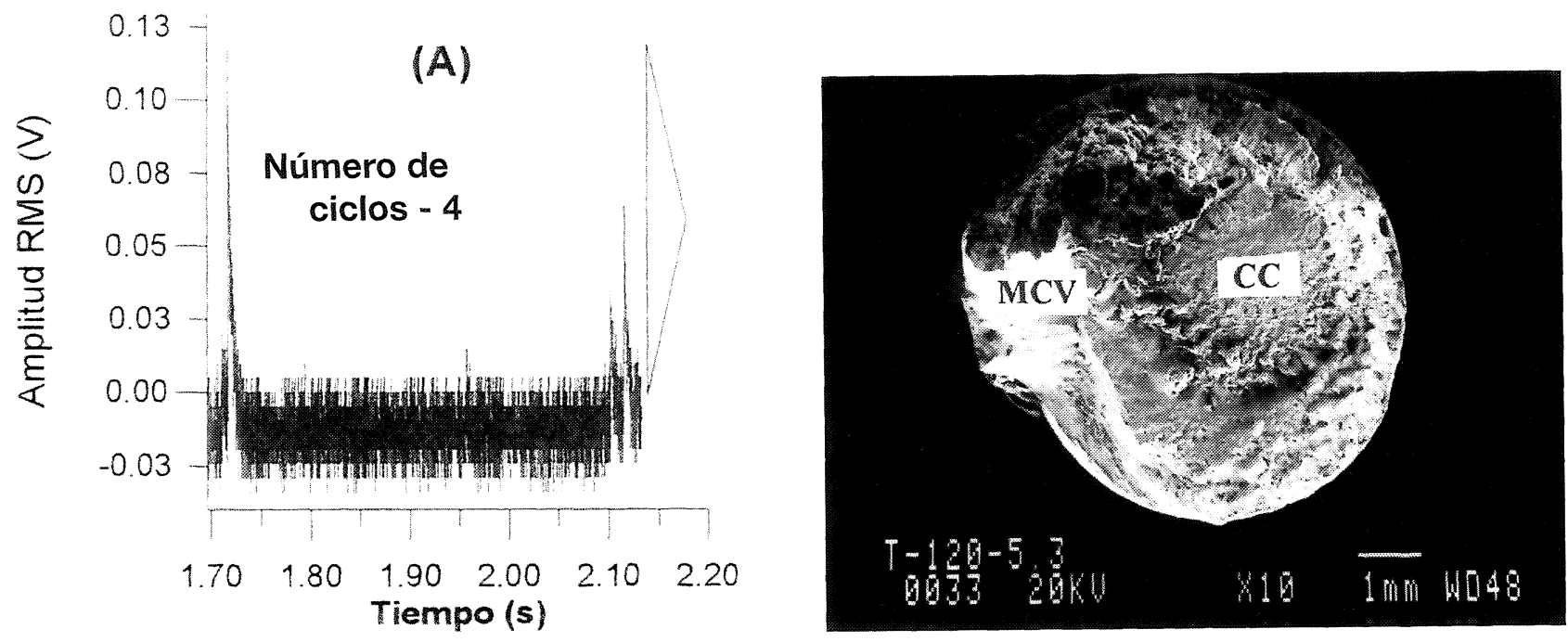

FIG. 5.- (a) Señal de EA instantes antes de la fractura final del implante con tensión aplicada de 580 MPa. (b) Vista general en el MEB de la superficie del implante fracturado.

FIG. 5.- (a) AE signal instant before implant fracture (580 MPa). (b) SEM view of a fractured implant specimen.
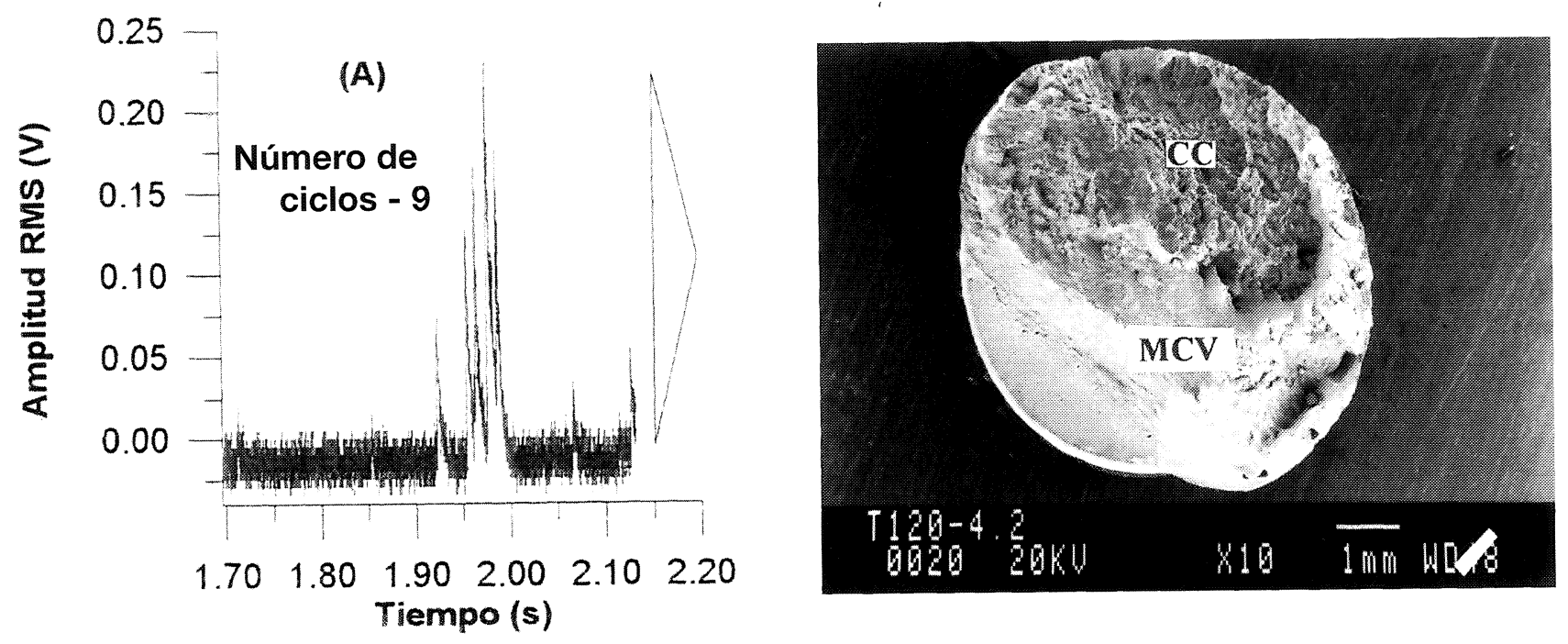

FIG. 6.- (a) Señal de EA instantes antes de la fractura final del implante con tensión aplicada de $457 \mathrm{MPa}$. (b) Vista general en el MEB de la superficie del implante fracturado.

FIG. 6. - (a) AE signal instant before implant fracture (457 MPa). (b) SEM view of a fractured implant specimen.

\section{CONCLUSIONES}

Los resultados experimentales mostraron que:

- La modificación producida para la realización del ensayo de implante en la zona fundida permitió la obtención de resultados coherentes, siendo de gran utilidad en el desarrollo de la investigación.

- La mezcla de $\mathrm{CO}_{2}+5 \% \mathrm{H}_{2}$, usada como gas de protección, posibilitó la obtención de altos niveles de hidrógeno difusible en la unión soldada.
- El alambre tubular T-120 fue más susceptible al agrietamiento por hidrógeno que el alambre T75 , lo que fue provocado por las características microestructurales y dureza del depósito.

- El modo de fractura mixto fue típico de la fractura en la zona fundida de los experimentos realizados con el alambre T-120. Se comprobó que para elevados niveles de hidrógeno difusible, el modelo de microplasticidad de Beachem (3) debe ser analizado más detalladamente.

- Los resultados experimentales mostraron que existe una importante dependencia entre los 


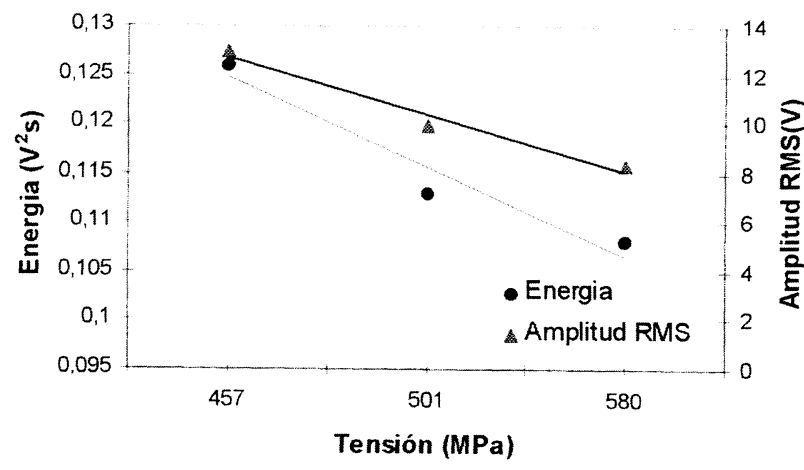

FIG. 7.- Correlación entre la energía y amplitud RMS de la señal de EA y la tensión aplicada.

FIG. 7.- RMS energy and amplitude as a function of the applied stress.

modos de fractura y las características de la señal de emisión acústica.

- Se determinaron los valores del número de ciclos, amplitud RMS y energía total de la señal de EA, características del fenómeno de agrietamiento asistido por hidrógeno en la zona fundida, lo que puede ser de gran validez para futuras investigaciones y aplicaciones industriales.

\section{Agradecimientos}

Los autores de este trabajo agradecen a la Fundación de Amparo a Pesquisa del Estado de Sao Paulo (FAPESP), el soporte financiero para la realización del proyecto de investigación.

\section{REFERENCIAS}

(1) Vuick, J., Weld. World, V31 (5), 1992: 308-321.

(2) Svensson, L.E. Control of Microestrutures and Properties in Steel arc Welds. The ESAB Group. Esab AB. Gotherburg (Suecia). CRC Pres, Inc., 1994: 39-44.

(3) BEACHEM, C.D. Metall. Trans., 3 (2), 1972: 437-451.

(4) Yurioka. N. y Suzuki. H. Int. Mater. Rev., 35 (4), 1990: 217-249.

(5) Gedeon, S.A. y Eagar, T.W; Weld. J., junio 1990: 213s.

(6) Fang, C.K., Kannatey-Asibu Jr, E. y Barber, J.R. Weld. J., junio 1995: 177-184.

(7) Trevisan, R.E. y Ferraresi, A.V. Abstracts of Papers. 79th Am. Weld. Soc. Annual Meeting (EE.UU.), 26-30 abril 1998:151.

(8) Carpenter, S.H. y Smith Jr, D.R. Metall. Trans. A, V 21A, julio 1990: 1933-1938.

(9) Fals, H.C. y Trevisan, R.E. Proceedings. 17th Int. Offshore، \& Arctic Engineering Conference. Lisboa (Portugal), 5-9 julio, 1998.

(10) American National Standards Institute/American Welding Society. EU, ANSI/AWS A 4-3-86. Standard Methods for Determination of the Diffusible Hydrogen Content of Martensitic, Bainitic, and Ferritic Steel Weld Metal Produced by Arc Welding, 1986: $17 \mathrm{p}$.

(11) NF A 89-100. Cold Cracking Test Methods Using Implants. Abril 1982: $10 \mathrm{p}$.

(12) Mota, J.M.F y APPS, R.L, Weld. J., julio 1982: 222s. 